\title{
Extracellular secretion in Bacillus subtilis of a cytoplasmic thermostable $\beta$-galactosidase from Geobacillus stearothermophilus
}

\author{
Y. Xia, ${ }^{*}$ J. Zhao, ${ }^{*}$ H. Chen, ${ }^{*}$ X. Liu, ${ }^{*}$ Y. Wang, ${ }^{*}$ F. Tian, ${ }^{*}$ H. P. Zhang,† H. Zhang, ${ }^{*}$ and W. Chen*1 \\ *State Key Laboratory of Food Science and Technology, School of Food Science and Technology, Jiangnan University, Wuxi 214122, P. R. China \\ †Key Laboratory of Dairy Biotechnology and Engineering, Ministry of Education, Inner Mongolia Agricultural University, Huhhot, Inner Mongolia \\ 010018, P. R. China
}

\begin{abstract}
$\beta$-Galactosidase catalyzes the hydrolysis of $\beta$-galactosides into monosaccharides and is widely used in dairy processing. This study reports the extracellular secretion of a cytoplasmic thermostable $\beta$-galactosidase from Geobacillus stearothermophilus IAM11001 in Bacillus subtilis. This enzyme has potential applications in the dairy industry. It was not secreted in B. subtilis by mediation of 3 general secretory signal peptides, but was secreted extracellularly when it was fused to a twin-arginine signal peptide of $B$. subtilis phosphodiesterase. Defined and rich culture media were used for recombinant enzyme production, and the extracellular target enzymatic activity reached about $44 \%$ of the total enzymatic activity synthesized at $18 \mathrm{~h}$ of cultivation in Luria-Bertani medium. As a control of secretion, when the signal peptide coding sequence was absent from the $\mathrm{N}$ terminus of the target gene bgaB, the extracellular target enzymatic activity obtained under the same condition of cultivation accounted for less than $7 \%$ of the total enzymatic activity synthesized. Results also showed that coexpression of the B. subtilis proteins TatAd and TatCd was indispensable for the secretion of the target enzyme.
\end{abstract}

Key words: Bacillus subtilis, secretion, thermostable $\beta$-galactosidase

\section{INTRODUCTION}

$\beta$-Galactosidase ( $\beta$-D-galactosidase; $\quad \mathbf{B g a B} ; \quad$ EC $3.2 .1 .23)$ catalyzes the hydrolysis of $\beta$-galactosides into monosaccharides and is widely used in dairy processing to eliminate lactose from milk. On an industrial scale, the $\beta$-galactosidases used in dairy processing are mainly mesophilic enzymes from Kluyveromyces and Aspergillus; thus, the lactose hydrolysis treatments are usually carried out at moderate temperatures, which might cause undesired microbial contaminations during

Received October 29, 2009.

Accepted March 8, 2010.

${ }^{1}$ Corresponding author: weichen@jiangnan.edu.cn the time of processing (Wanarska et al., 2005). To overcome this problem, thermostable BgaB might be used as alternatives because they can work at high temperatures (Chen et al., 2008). We have previously reported the recombinant production of a thermostable $\mathrm{BgaB}$ from Geobacillus stearothermophilus IAM11001 (Hirata et al., 1984) in Bacillus subtilis, as well as the purification and characterization of this enzyme (Chen et al., 2008). At pH 7.0, this recombinant enzyme exhibited the maximum enzymatic activity at $70^{\circ} \mathrm{C}$, and $80 \%$ of the enzymatic activity was still observed at $75^{\circ} \mathrm{C}$. Besides, this enzyme has a high level of transgalactosylation activity for production of galacto-oligosaccharides. Because G. stearothermophilus is generally recognized as safe for production of food additives (Gote et al., 2004), the thermostable BgaB derived from this strain is an ideal candidate for dairy processing.

In our previous study (Chen et al., 2008), the final enzymatic activity of the thermostable BgaB was much higher than that obtained from the original strain G. stearothermophilus IAM11001, yet this enzyme is cytoplasmic and the recombinant production strains needed sonication to release the target enzyme (Hirata et al., 1985) and multistep procedures were needed for purification of this enzyme if it was intended for future industrial use. Bacillus subtilis naturally has high capacity of protein secretion and is historically used as a selected host for secretory production of industrial proteins (Tjalsma et al., 2000), so it is worth investigating whether this enzyme can be secreted by $B$. subtilis to the extracellular medium so that the downstream purification procedures of the enzyme might be simplified. This cytoplasmic enzyme has never been reported secreted, so investigation of the secretion capacity of this enzyme is of theoretical interest. Bacillus subtilis has at least 4 distinct protein secretion pathways, of which the general secretion (Sec) pathway is the major pathway used for industrial purpose (Tjalsma et al., 2004). The twin-arginine translocation (Tat) pathway is the second general protein Sec pathway in most bacteria (Berks et al., 2000). Because the Tat pathway can secrete cytoplasmically folded proteins (Tjalsma et 
al., 2004; Barnett et al., 2009), it has potential for and ought to be applied to the secretion of Sec-incompatible heterologous proteins (van Dijl et al., 2002; Kikuchi et al., 2009). In this study, we attempted to secrete the cytoplasmic thermostable BgaB mentioned above in $B$. Subtilis with both Sec and Tat type signal peptides.

\section{MATERIALS AND METHODS}

\section{Bacterial Strains and Plasmids and Reagents}

Bacterial strains and plasmids used in this work are listed in Table 1. Restriction enzymes and molecular weight protein marker were purchased from MBI Fermentas (Shanghai, China), KOD plus DNA polymerase was purchased from Toyobo (Shanghai, China) and was used in all PCR in this work, PCR Purification Mini Kit and Gel Extraction Mini Kit were purchased from Watson Biotechnology (Shanghai, China), and agar powder was purchased from Sibas Bio-tech (Shanghai, China). Other reagents were of analytical reagent grade.

\section{Media and Culture Conditions}

Escherichia coli and B. subtilis strains were grown at $37^{\circ} \mathrm{C}$, Bacillus megaterium was grown at $30^{\circ} \mathrm{C}$, and $G$. stearothermophilus was grown at $55^{\circ} \mathrm{C}$. All the cultures were grown in Luria-Bertani (LB) liquid medium or on LB agar plates as needed. Low-phosphate defined medium (LPDM) and high-phosphate defined medium (HPDM) were especially prepared for phosphate-starvation inducible expression of target enzyme according to the method published by Müller et al. (1997). Ampicillin $(100 \mu \mathrm{g} / \mathrm{mL})$, chloramphenicol $(5 \mu \mathrm{g} / \mathrm{mL})$, and kanamycin $(50 \mu \mathrm{g} / \mathrm{mL})$ were added to LB medium as needed.

\section{Genetic Manipulations}

Manipulations of DNA including purification, restriction digestion, ligation, agarose gel electrophoresis, PCR, and other genetic manipulations in E. coli were performed as described by Sambrook and Russell (2001), whereas those in Bacillus were performed as described by Harwood and Cutting (1990). Primers used for PCR are listed in Table 2. All the amplified fragments were sequenced by Invitrogen (Shanghai, China) before molecular cloning.

\section{Construction of Plasmids for Sec Secretion}

The signal peptide coding sequence of $B$. subtilis $\alpha$-amylase was amplified from the chromosome of $B$. subtilis 168 (Kunst et al., 1997) by PCR with the prim- ers $\mathrm{P} 1$ and $\mathrm{P} 2$. This fragment was digested with restriction endonucleases NdeI and BamHI and then cloned to an E. coli-B. subtilis shuttle vector pMA5 (Westers et al., 2006) to generate plasmid pMsig01. The signal peptide coding sequence of $B$. subtilis neutral protease $\mathrm{NprB}$ was amplified from the chromosome of B. subtilis 168 by PCR with the primers P 3 and P4. This fragment was digested with $\mathrm{NdeI}$ and $\mathrm{BamHI}$ and then cloned to pMA5 to generate plasmid pMsig02. The signal peptide coding sequence of penicillin $\mathrm{G}$ acylase was amplified from the chromosome of B. megaterium ATCC14945 (Kang et al., 1994) by PCR with the primers P5 and P6. This fragment was also digested with NdeI and $B a m \mathrm{HI}$, and then cloned to pMA5 to generate plasmid pMsig03. The thermostable BgaB coding sequence bgaB was amplified from the chromosome of $G$. stearothermophilus IAM11001 by PCR with the primers P7 and P8. This fragment was digested with BamHI and SacI and then cloned to the corresponding restriction sites of plasmids pMsig01, pMsig02, and pMsig03 to generate plasmids pMsig01-bgaB, pMsig02-bgaB, and pMsig03-bgaB, respectively.

The gene $b g a B$ was again amplified from the chromosome of G. stearothermophilus IAM11001 by PCR with the primers P9 and P8. This fragment was digested with NdeI and SacI and then cloned to the plasmid pMA5 to generate pMAbgaB. No signal peptide coding sequence was fused at the $5^{\prime}$-terminus of the $\mathrm{bgaB}$ sequence in pMAbgaB.

\section{Construction of Plasmids for Tat Secretion}

The promoter $\left(\mathbf{P}_{\mathbf{P h o D}}\right)$ and the signal peptide coding sequence of B. subtilis phosphodiesterase (PhoD) were amplified as a whole fragment from the chromosome of B. subtilis 168 by PCR with the primers $\mathrm{P} 10$ and $\mathrm{P} 11$. The gene $b g a B$ was amplified from the chromosome of G. stearothermophilus IAM11001 by PCR with the primers $\mathrm{P} 12$ and $\mathrm{P} 13$. The 2 fragments were spliced into a fusion fragment by overlap extension PCR with the primers $\mathrm{P} 10$ and $\mathrm{P} 13$. In this fusion fragment, the initiation codon (ATG) of gene bgaB was fused directly to the codon GCT of the signal peptide coding sequence. According to the published data (Eder et al., 1996), the codon GCT was located just upstream of the first codon (GCG) of PhoD mature peptide, so in this fusion the structural gene bgaB exactly replaced the PhoD mature peptide coding sequence. This fusion fragment was digested with BamHI and EcoRI and then cloned into the corresponding sites of plasmid pMK4 to generate plasmid pMsig04-bgaB.

This fusion fragment was again subjected to PCR amplification with the primers $\mathrm{P} 14$ and $\mathrm{P} 15$ to introduce a 5'-terminal $N d e I$ site and a 3 '-terminal BamHI site. 
Table 1. Bacterial strains and plasmids

Strain/plasmid

$$
\text { JM109 }
$$

Geobacillus stearothermophilus

$$
\begin{array}{r}
\text { Geobacillus } \\
\text { IAM11001 }
\end{array}
$$

Bacillus subtilis

168

Plasmids

pMA5

pMsig01

pMsig02

pMsig03

pMsig01-bgaB

pMsig02-bgaB

pMsig03-bgaB

pMAbgaB

pMK4

pMsig04-bgaB

pMKbgaB

pMsig05-bgaB

pMtat

pMtat-sigbgaB
Description

recA supE44 endA1 hsdR17 gyrA96relA1 this(lac-proAB) F'[traD36 proAB $B^{+}$lacI"lacZAM15];

used as host for plasmid construction

Source of the thermostable $\beta$-galactosidase

$\operatorname{trp} C 2$; used as source of sequences needed and host for expression

ColE1, repB, $\mathrm{Km}^{\mathrm{r}}, \mathrm{Ap}^{\mathrm{r}}, \mathrm{P}_{\text {HpaII }}$; E. coli-B. subtilis shuttle vector derived from plasmid pUB110

pMA5 derivative carrying the $\mathrm{SP}_{\mathrm{AmyE}_{3}}$

pMA5 derivative carrying the $\mathrm{SP}_{\mathrm{NprB}_{4}}{ }_{4}$

PMA5 derivative carrying the $\mathrm{SP}_{\mathrm{PGA}}$

pMsig01 derivative carrying the $\mathrm{P}_{\text {HpaII }}-\mathrm{SP}_{\mathrm{AmyE}}-$ bgaB fusion

pMsig02 derivative carrying the $\mathrm{P}_{\mathrm{HpaII}}-\mathrm{SP}_{\mathrm{Npr}^{-}}$bgaB fusion

pMsig03 derivative carrying the $\mathrm{P}_{H p a I I}-\mathrm{SP}_{\mathrm{PGA}}-b g a B$ fusion

pMA5 derivative carrying the $\mathrm{P}_{\text {HpaII }}-$ bgaB fusion; nonsecretory

$\mathrm{Amp}^{\mathrm{r}} \mathrm{Cm}^{\mathrm{r}} \mathrm{Lac}^{+} ; E$. coli-B. subtilis shuttle vector derived from plasmid $\mathrm{pC} 194$

pMK4 derivative carrying the $\mathrm{P}_{\mathrm{PhoD}^{-}} \mathrm{SP}_{\mathrm{PhoD}^{-}}$bgaB fusion

pMK4 derivative carrying the $\mathrm{P}_{\mathrm{PhoD}^{-}}-\mathrm{bgaB}$ fusion; nonsecretory

pMA5 derivative carrying the $\mathrm{P}_{H p a I I}-\mathrm{SP}_{\mathrm{PhoD}^{-}}-b g a B$ fusion

pMA5 derivative carrying the $\mathrm{P}_{H p a I}$-tatAd-tatCd fusion

pMA5 derivative carrying the $\mathrm{P}_{H}$-tatAd-tatCd fusion, $\mathrm{SP}_{\mathrm{PhoD}^{-}-b g a B}$ fusion

${ }^{1}$ This laboratory $=$ State Key Laboratory of Food Science and Technology, School of Food Science and Technology, Jiangnan University, Wuxi, P. R. China; BGSC = Bacillus Genetic Stock Center, The Ohio State University, Columbus.

${ }^{2} \mathrm{SP}_{\mathrm{AmyE}}$ : signal peptide coding sequence of $B$. subtilis $168 \alpha$-amylase.

${ }^{3} \mathrm{SP}_{\mathrm{NprB}}$ : signal peptide coding sequence of B. subtilis 168 neutral protease.

${ }^{4} \mathrm{SP}_{\mathrm{PGA}_{\mathrm{A}}}$ : signal peptide coding sequence of B. megaterium ATCC14945 penicillin G acylase. 
Table 2. Primers used in this work

\begin{tabular}{ll}
\hline Primer & Sequence $^{1}$ \\
\hline P1 & 5'-GGGCCCGGCATATGTTTGCAAAACGATTCAAAACC-3' \\
P2 & 5'-AATTTAGGATCCAGCACTCGCAGCCGCCGGT-3' \\
P3 & 5'-CGTCGTGCATATGCGCAACTTGACCAAGACATCTC-3' \\
P4 & 5'-GAGCGGGGATCCAGCTGAGGCATGTGTTACAA-3' \\
P5 & 5'-GGGCCATATGAAGACGAAGTGGCTAAT-3' \\
P6 & 5'-GGCCGGATCCATTCTTATCATTCTTATCCTC-3' \\
P7 & 5'-GGCCGAATCCATGAATGTGTTATCCTCAAT-3' \\
P8 & 5'-GGCCGAGCTCCTAAACCTTCCCGGCTTCAT-3' \\
P9 & 5'-GGCCCATATGAATGTGTTATCCTCAAT-3' \\
P10 & 5'-GGGCCCGGATCCGTAAGAGAACAAGAGCCTCC-3' \\
P11 & 5'-TAACACATTCATAGCATTTACTTCAAAGGCCCC-3' \\
p12 & 5'-GAAGTAAATGCTATGAATGTGTTATCCTCAA-3' \\
p13 & 5'-GGCCCGAATTCGATCCAATGTTTATAGACA-3' \\
P14 & 5'-GGGGGGCATATGGCATACGACAGTCGTTTT-3' \\
P15 & 5'-GGCCGGATCCCTAAACCTTCCCGGCTTCAT-3' \\
P16 & 5'-GGGCCCCATATGTTTTCAAACATTGGAATAC-3' \\
P17 & 5'-AATATTGGATCCGTGGTACGCTGCACTAAGCG-3' \\
P18 & 5'-GGCCGGATCCAAAGGAGCGATTTAATGTTTTCAAACATTGGAA-3' \\
P19 & 5'-GGCCGAGCTCCTAAACCTTCCCGGCTTCAT-3' \\
P20 & 5'-GAAAAACCCGGGAGTTCTAGGGGAGAGAGGAGT-3' \\
P21 & 5'-GCCAAACCCGGGATGAATGTGTTATCCTCAATTTG-3' \\
\hline
\end{tabular}

${ }^{1}$ The restriction sites are underlined and the regions used for overlap extension are in bold.

The amplified fragment was digested with $N d e \mathrm{I}$ and BamHI and then cloned into the corresponding sites of pMA5. The resultant plasmid was named pMsig05bgaB, in which the signal peptide coding sequence- $b g a B$ fusion (SP-bgaB) was under control of the constitutive promoter $\mathrm{P}_{\text {HpaII }}$. The TatAd/Cd coding sequence tatAd/ $C d$ was amplified from the chromosome of $B$. subtilis by PCR using primers P16 and P17. This fragment was digested with $N d e \mathrm{I}$ and $B a m \mathrm{HI}$ and then cloned to the corresponding sites of pMA5 to generate pMtat. The SP-bgaB fusion was amplified by PCR using plasmid pMsig05-bgaB as a template and the primers P18 and P19. This fragment was digested with BamHI and SacI and then cloned to the corresponding sites of plasmid pMtat to generate pMtat-sigbgaB.

Plasmid pMsig04-bgaB was subjected to PCR amplification with the primers P20 and P21. The amplified fragment was digested with $S m a \mathrm{I}$ and self-ligated to generate the plasmid $\mathrm{pMKbgaB}$, in which the gene bgaB was under control of promoter $\mathrm{P}_{\mathrm{PhoD}}$ and the signal peptide coding sequence was absent from the 5 '-terminus of this gene.

\section{Enzymatic Assays}

Cell preparation, sonication, enzymatic assays, and SDS-PAGE analysis of the thermostable $\beta$-galactosidase were performed as described previously (Xia et al., 2005). One unit of enzymatic activity was defined as the amount of enzyme that hydrolyzes $1 \mathrm{nmol}$ of substrate (o-nitrophenyl- $\beta$-D-galactopyranoside) per minute according to Yuan and Wong (1995).

\section{RESULTS}

\section{Secretion of Thermostable $\beta$-Galactosidase with the Sec Signal Peptides}

On an industrial scale, heterologous proteins are secreted mainly by the Sec pathway. In this experiment, 3 Sec signal peptides were employed for secretion of the thermostable BgaB. The secretion plasmids pMsig01-bgaB, pMsig02-bgaB, and pMsig03-bgaB were constructed for secretion test of $\mathrm{BgaB}$, in which the signal peptide coding sequences of $B$. subtilis $\alpha$-amylase, neutral protease, and B. megaterium penicillin $G$ acylase were fused to the $5^{\prime}$-terminus of gene $b \mathrm{gaB}$. As a negative control of Sec secretion, the plasmid pMAbgaB was constructed for intracellular expression of $\mathrm{BgaB}$, in which the signal peptide coding sequence was absent from the 5 '-terminus of gene $b g a B$. The 4 plasmids were transformed in the host strain, B. subtilis 168 , and yielded recombinant strains 168 (pMsig01bgaB), 168(pMsig02-bgaB), 168(pMsig03-bgaB), and 168(pMAbgaB), respectively.

The 4 strains were each cultivated in LB liquid at $37^{\circ} \mathrm{C}$. After $8 \mathrm{~h}$ of cultivation, aliquots were taken out every hour from the cultures for investigation of the intracellular and extracellular (supernatant) BgaB enzymatic activities. The intracellular enzymatic activities of the 4 strains were close to each other, and the extracellular enzymatic activities were close to each other as well. Using the strain 168(pMsig01-bgaB) as an example, at $18 \mathrm{~h}$ of cultivation the extracellular enzymatic activity reached only about $173.6 \mathrm{U} / \mathrm{mL}$, whereas the intracel- 

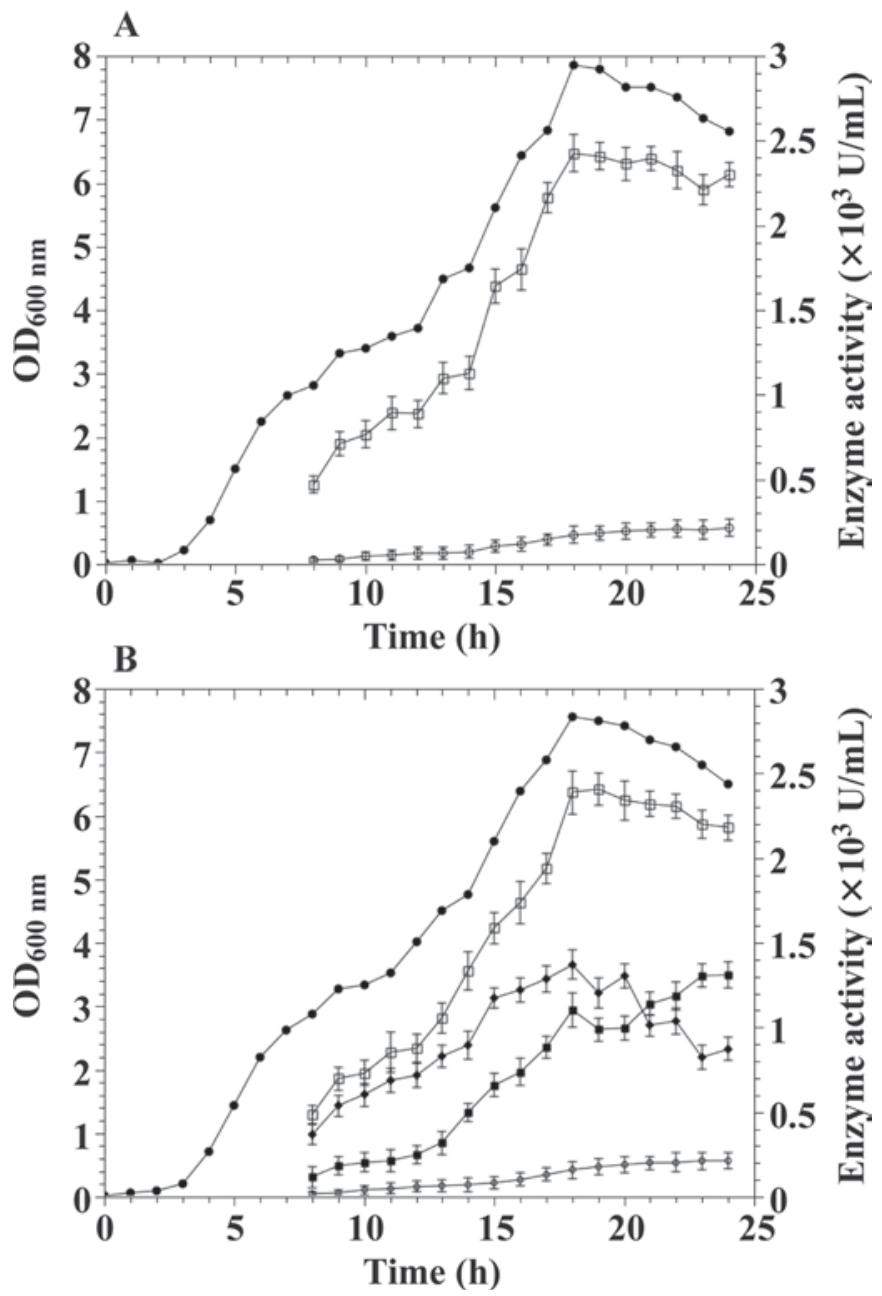

Figure 1. Growth curve and $\beta$-galactosidase (BgaB) enzymatic activities of A) 168(pMsig01-bgaB) and B) 168(pMtat-sigbgaB), 168(pMsig05-bgaB). Overnight, primary cultures were inoculated (1\%) into $100 \mathrm{~mL}$ of Luria-Bertani liquid medium supplemented with kanamycin for cultivation at $37^{\circ} \mathrm{C}$ with shaking at $200 \mathrm{rpm}$. Aliquots were taken at different time points of cultivation to determine the growth curve. The intracellular and extracellular BgaB enzymatic activities were determined from $8 \mathrm{~h}$ of cultivation. The enzymatic activities were the average of 3 independent determinations and given as mean \pm SD. A) Growth of 168(pMsig01-bgaB) (-); intracellular BgaB enzymatic activity of 168 (pMsig01-bgaB) ( $\square$ ); extracellular BgaB enzymatic activity of 168(pMsig01-bgaB) (O). B) Growth of 168(pMtat-sigbgaB) $(\bullet)$; intracellular BgaB enzymatic activity of 168(pMtat-sigbgaB) ( ); extracellular BgaB enzymatic activity of 168(pMtat-sigbgaB) (ם); intracellular BgaB enzymatic activity of 168(pMsig05-bgaB) ( $\square$ ); extracellular BgaB enzymatic activity of 168(pMsig05-bgaB) (O). OD = optical density.

lular enzymatic activity reached about $2,426.0 \mathrm{U} / \mathrm{mL}$ (Figure 1A). This result showed that less than $7 \%$ of the total enzymatic activity synthesized existed in the supernatant. Because the extracellular BgaB activities produced by pMsig01-bgaB, pMsig02-bgaB, and pMsig03-bgaB were both low $(<7 \%)$ and close to that obtained from pMAbgaB (no signal peptide coding sequence used; Table 3), it indicated that the extracel- lular BgaB produced by the 3 plasmids with the Sec signal peptide coding sequence was obtained from cell autolysis rather than secretion.

\section{Secretion of Thermostable $\beta$-Galactosidase with the Tat Signal Peptide in Defined Media}

In B. subtilis, the phosphodiesterase PhoD is a strictly Tat-dependent secretion protein (Tjalsma et al., 2004). Here, the original promoter $\left(\mathrm{P}_{\mathrm{PhoD}}\right)$ and the signal peptide coding sequence of $\mathrm{PhoD}$ were used for secretion test of the target enzyme. The secretory expression plasmid pMsig04-bgaB carrying the $\mathrm{P}_{\mathrm{PhoD}^{-}}-\mathrm{SP}-$ bgaB fusion was constructed and used to transform $B$. subtilis 168 , and thus yielded 168(pMsig04-bgaB). Because the promoter $\mathrm{P}_{\mathrm{PhoD}}$ is phosphate-starvation inducible and is regulated by the 2-component signal transduction system PhoP-PhoR, expression of BgaB by pMsig04bgaB was carried out in LPDM according to Eder et al. (1996). As a control of expression, this experiment was repeated in HPDM, in which the transcription function of $\mathrm{P}_{\mathrm{PhoD}}$ should be inhibited. At $18 \mathrm{~h}$ of cultivation, the extracellular BgaB enzymatic activity obtained from 168(pMsig04-bgaB) in LPDM was $80.1 \mathrm{U} / \mathrm{mL}$, which accounted for $30 \%$ of the total BgaB enzymatic activity obtained, whereas in HPDM, neither the extracellular nor the total BgaB enzymatic activities were detected (Table 3). As a control of secretion, the plasmid pMKbgaB, which had no signal peptide coding sequence, was constructed and used to transform 168 for expression of BgaB in LPDM, but no extracellular BgaB enzymatic activity was detected. Meanwhile, the total BgaB enzymatic activity obtained from 168(pMsig04-bgaB) in LPDM was $267.3 \mathrm{U} / \mathrm{mL}$, similar to the total activity obtained from 168(pMKbgaB) in LPDM (Table 3). These results indicated that the promoter $\mathrm{P}_{\mathrm{PhoD}}$ was induced by the phosphate-starvation medium and the $\mathrm{BgaB}$ was secreted in LPDM by mediation of PhoD signal peptide.

\section{Secretion of Thermostable $\beta$-Galactosidase with the Tat Signal Peptide in Rich Medium}

Although the extracellular enzymatic activity expressed by 168(pMsig04-bgaB) in LPDM was obvious, it was still low because of the poor growth of the recombinant strain in defined medium. To get higher secretion level of this enzyme, secretory expression was tried in rich medium LB. The B. subtilis proteins TatAd and TatCd, which form the Tat translocase TatAdCd for Tat-dependent protein secretion, were expressed only in low-phosphate containing medium (Jongbloed et al., 2000). In this experiment, for expression of TatAd and TatCd in LB, the genes tatAd/Cd were cloned under the 
Table 3. $\beta$-Galactosidase (BgaB) enzymatic activities obtained from different strains at $18 \mathrm{~h}$ of cultivation ${ }^{1}$

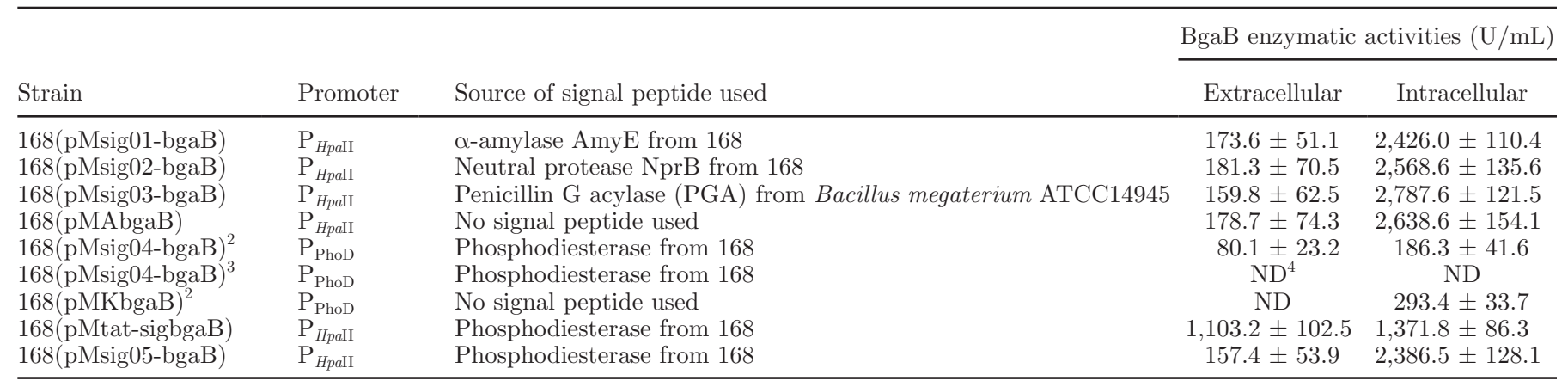

${ }^{1}$ Overnight, primary cultures of different recombinant strains were inoculated (1\%) into $100 \mathrm{~mL}$ of Luria-Bertani liquid medium supplemented with antibiotics for cultivation at $37^{\circ} \mathrm{C}$ with shaking at $200 \mathrm{rpm}$. Aliquots were taken at $18 \mathrm{~h}$ of cultivation (late-logarithmic growth phase) to determine the extracellular and total BgaB enzymatic activities. The values were the average of 3 independent determinations and given as mean \pm SD.

${ }^{2}$ Expression was carried out in low-phosphate defined medium.

${ }^{3}$ Expression was carried out in high-phosphate defined medium.

${ }^{4} \mathrm{ND}=$ not detected.

control of the constitutive promoter $\mathrm{P}_{H p a I I}$ in plasmid pMA5 and yielded plasmid pMtat. The SP-bgaB fusion was cloned downstream of $t a t A d / C d$ expression unit in pMtat and yielded the plasmid pMtat-sigbgaB. Plasmid pMsig05-bgaB was constructed and used for negative control of secretion, in which the tatAd/Cd sequence was absent from the upstream of the SP-bgaB fusion. In the plasmids pMsig05-bgaB and pMtat-sigbgaB, the genes bgaB and tatAd/Cd had their own but the same ribosome binding site sequences (5'-AAAGGAGCG-3'), and the 2 ribosome binding sites were located upstream of the initiation codons ATG.

After transformation of the 2 plasmids to 168 , the resultant strains 168(pMtat-SigbgaB) and 168(pMsig05bgaB) were cultivated in LB liquid for expression and secretion of BgaB. The growth curve of the recombinant strain 168(pMtat-SigbgaB) and the extracellular and intracellular enzymatic activities obtained from 168(pMtat-SigbgaB) and 168(pMsig05-bgaB) were examined (Figure 1B). During the cultivation course of 8 to $18 \mathrm{~h}$, the extracellular BgaB enzymatic activities from 168(pMtat-SigbgaB) at every time point accounted for at least $24 \%$ of their corresponding total activities. At $18 \mathrm{~h}$ of cultivation, the extracellular enzymatic activity from 168(pMtat-SigbgaB) reached about 1,103.2 U/mL (Table 3), which accounted for more than $44 \%$ of the total BgaB activity obtained and was 13 times higher than that obtained from 168(pMsig04-bgaB) in LPDM. At every time point, the total BgaB activity from 168(pMsig05-bgaB) was close to that obtained from 168(pMtat-SigbgaB), whereas the extracellular BgaB activity from 168(pMsig05-bgaB) was kept at a low level. For example, at $18 \mathrm{~h}$ of cultivation, the extracellular BgaB activity obtained from 168(pMsig05-bgaB) was $157.4 \mathrm{U} / \mathrm{mL}$, which accounted for only $6.2 \%$ of the total activity.

The cultivation supernatants and cell-free extracts of 168(pMtat-sigbgaB) and 168 (pMsig05-bgaB) were subjected to SDS-PAGE analysis. Results showed the extracellular BgaB band in the supernatant of 168(pMtat-sigbgaB) culture was obvious, whereas it was difficult to show the extracellular BgaB band in the supernatant of 168(pMsig05-bgaB) cultures (Figure 2 ). These results indicated that the major extracellular BgaB activity in the cultivation supernatant of 168(pMtat-sigbgaB) was obtained by secretion rather than cell autolysis.

\section{DISCUSSION}

Thermostable $\beta$-galactosidase has potential applications in dairy industry. In this work, the heterologous cytoplasmic thermostable $\beta$-galactosidase from $G$. stearothermophilus IAM11001 was a target enzyme to be secreted in B. subtilis. Because the Sec pathway is used in industry mainly for extracellular production of proteins, we first tried 3 Sec signal peptides in this work. Results showed that the target enzyme BgaB was expressed successfully, but the extracellular target enzymatic activities obtained from the secretory plasmids (pMsig01-bgaB, pMsig02-bgaB, and pMsig03-bgaB) were at a relatively low level and close to that from the nonsecretory plasmid pMAbgaB. Because cell autolysis is a normal phenomena essential for cell growth of $B$. subtilis (Smith et al., 2000), the low level of extracellular enzymatic activities obtained should have resulted from partial cell autolysis rather than Sec secretion. The Sec pathway was frequently reported inefficient or 
even impracticable for secretion of heterologous proteins because of a variety of bottlenecks in different stages of translocation (van Dijl et al., 2002; Li et al., 2004). One potential bottleneck lies in the incompatibility between target proteins and the Sec translocases. Proteins that fold before translocation are usually not able to be secreted by the Sec pathway (Gerlach et al., 2004). In this work, major parts of the recombinant thermostable $\beta$-galactosidases synthesized were located intracellularly, which also indicated that this enzyme was folded properly in the cytoplasm. This natural property might be responsible for the unsuccessful secretion of the enzyme by the Sec signal peptides, and it prompted us to try the Tat signal peptide because the Tat pathway can export folded proteins. So far, several heterologous proteins had been secreted by Tat signal peptides in E. coli or B. subtilis (Brüser, 2007; Kolkman et al., 2008), whereas the phosphodiesterase PhoD is secreted in a strictly Tat-dependent manner in B. subtilis (Tjalsma et al., 2004). Therefore, the signal peptide of PhoD was employed for Tat secretion of the target enzyme in this work.

Expression and secretion of the target enzyme were carried out in defined media to determine whether the original expression and secretion elements of PhoD (the phosphate-starvation inducible promoter, the signal peptide sequence, and the translocase TatAdCd) can direct the secretion of the target enzyme. The comparative results obtained from LPDM and HPDM confirmed that these elements of PhoD did mediate the secretion process, but in a relatively low level because of insufficient growth of the recombinant strain in the defined medium. Secretion of the target enzyme was then carried out in rich medium LB with strong constitutive promoter and coexpression of the Tat translocase elements TatAd and TatCd. The supernatant enzymatic activities from 168(pMtat-sigbgaB) was much higher than those obtained from 168 (pMsig05-bgaB), indicating that coexpression of genes $t a t A d / C d$ is indispensible for export of the target enzyme. Meanwhile, the existence of intracellular BgaB activity (more than $50 \%$ of the total activity) revealed that cytoplasmic folding of the BgaB precursor ahead of the translocation process occurred, which was regarded as an essential determinant for Tat-dependent protein secretion in B. subtilis (Kolkman et al., 2008). However, cytoplasmic folding of this enzyme and employment of the PhoD signal peptide were still not sufficient to determine the strictly Tat-dependent secretion of the substrate because there was exception; for example, the B. subtilis subtilisin was reported to be secreted Tat-independently in B. subtilis by mediation of the PhoD signal peptide (Kolkman et al., 2008). Further experiments should be conducted to confirm the secretion type of the target enzyme.

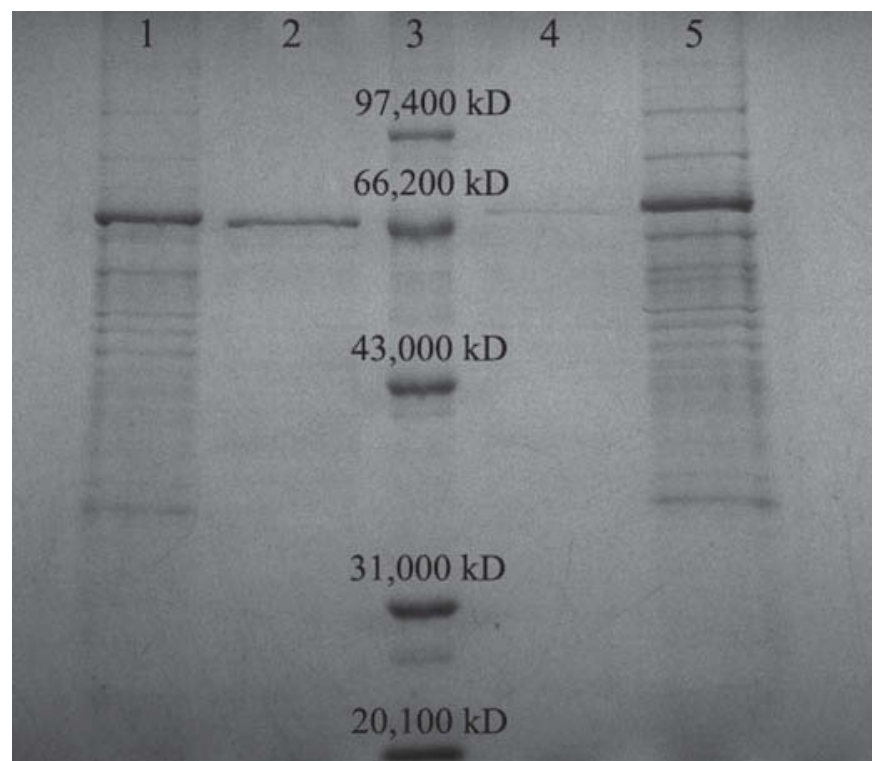

Figure 2. Sodium dodecyl sulfate-PAGE of cell-free extracts and cultivation supernatants of 168 (pMtat-sigbgaB) and 168(pMsig05bgaB). The electrophoresis was performed on a $10 \%$ polyacrylamide gel. Molecular weight protein marker (lane 3) was loaded with $10 \mu \mathrm{L}$ of sample, whereas lanes $1,2,4$, and 5 were loaded with $6 \mu \mathrm{L}$ of sample. Lane 1: cell-free extracts of 168(pMtat-sigbgaB). Lane 2: cultivation supernatant of 168 (pMtat-sigbgaB) (the mature protein showed a molecular weight of about $70 \mathrm{kD}$ ). Lane 3: molecular weight protein marker (the molecular weight was marked above the band). Lane 4: cultivation supernatant of 168 (pMsig05-bgaB) (negative control; a very slight band was shown that might be caused by cell autolysis). Lane 5: cell-free extracts of 168(pMsig05-bgaB).

On the other hand, the extracellular BgaB activity obtained from 168(pMtat-sigbgaB) in 8 to $18 \mathrm{~h}$ of cultivation accounted for no more than $50 \%$ of the enzymatic activity synthesized. This result was consistent with the previously reported low secretion levels of heterologous proteins by the Tat pathway in both E. coli (Barrett et al., 2003) and B. subtilis (Pop et al., 2002). Fortunately, this drawback might be alleviated by coexpression of cellular factors (DeLisa et al., 2004; Jongbloed et al., 2004) or by optimization of the signal peptide cleavage site (Gerlach et al., 2004). Additionally, other B. subtilis Tat signal peptides can also be tried for secretion of this enzyme.

\section{ACKNOWLEDGMENTS}

We thank the Bacillus Genetic Stock Center at The Ohio State University (Columbus) for providing the strains needed. This work was supported by the National Natural Science Foundation of China (Beijing) under Grant No. 30670065, the National High Technology Research and Development Program of China (Beijing) under Grant No. 2006AA102318 and 2007AA10Z316, The National Research Foundation for the Doctoral Program of Higher Education of China (Beijing) under 
Grant No. 200802951022, and the Science and Nature Foundation in Jiangsu Province (Nanjing, China) under Grant No. BK2008103).

\section{REFERENCES}

Barnett, J. P., R. van der Ploeg, R. T. Eijlander, A. Nenninger, S. Mendel, R. Rozeboom, O. P. Kuipers, J. M. van Dijl, and C. Robinson. 2009. The twin-arginine translocation (Tat) systems from Bacillus subtilis display a conserved mode of complex organization and similar substrate recognition requirements. FEBS J. 276:232-243.

Barrett, C. M., N. Ray, J. D. Thomas, C. Robinson, and A. Bolhuis. 2003. Quantitative export of a reporter protein, GFP, by the twinarginine translocation pathway in Escherichia coli. Biochem. Biophys. Res. Commun. 304:279-284.

Berks, B. C., F. Sargent, and T. Palmer. 2000. The Tat protein export pathway. Mol. Microbiol. 35:260-274.

Brüser, T. 2007. The twin-arginine translocation system and its capability for protein secretion in biotechnological protein production. Appl. Microbiol. Biotechnol. 76:35-45.

Chen, W., H. Chen, Y. Xia, J. Zhao, F. Tian, and H. Zhang. 2008. Production, purification, and characterization of a potential thermostable galactosidase for milk lactose hydrolysis from Bacillus stearothermophilus. J. Dairy Sci. 91:1751-1758.

DeLisa, M. P., P. Lee, T. Palmer, and G. Georgiou. 2004. Phage shock protein PspA of Escherichia coli relieves saturation of protein export via the Tat pathway. J. Bacteriol. 186:366-373.

Eder, S., L. Shi, K. Jensen, K. Yamane, and F. M. Hulett. 1996. A Bacillus subtilis secreted phosphodiesterase/alkaline phosphatase is the product of a Pho regulon gene, phoD. Microbiol. 142:20412047.

Gerlach, R., O. Pop, and J. P. Müller. 2004. Tat dependent export of E. coli phytase AppA by using the PhoD-specific transport system of Bacillus subtilis. J. Basic Microbiol. 44:351-359.

Gote, M., H. Umalkar, I. Khan, and J. Khire. 2004. Thermostable $\alpha$-galactosidase from Bacillus stearothermophilus (NCIM 5146) and its application in the removal of flatulence causing factors from soymilk. Process Biochem. 39:1723-1729.

Harwood, C. R., and S. M. Cutting. 1990. Molecular Biological Methods for Bacillus. Wiley, Sussex, UK.

Hirata, H., S. Negoro, and H. Okada. 1984. Molecular basis of isozyme formation of $\beta$-galactosidases in Bacillus stearothermophilus: Isolation of two $\beta$-galactosidase genes, bga $A$ and $b g a B$. J. Bacteriol. 160:9-14.

Hirata, H., S. Negoro, and H. Okada. 1985. High production of thermostable $\beta$-galactosidase of Bacillus stearothermophilus in Bacillus subtilis. Appl. Environ. Microbiol. 49:1547-1549.

Jongbloed, J. D. H., U. Grieger, H. Antelmann, M. Hecker, R. Nijland, S. Bron, and J. M. van Dijl. 2004. Two minimal Tat translocases in Bacillus. Mol. Microbiol. 54:1319-1325.

Jongbloed, J. D. H., U. Martin, H. Antelmann, M. Hecker, H. Tjalsma, G. Venema, S. Bron, J. M. van Dijl, and J. Müller. 2000. TatC is a specificity determinant for protein secretion via the twin-arginine translocation pathway. J. Biol. Chem. 275:41350-41357.

Kang, J. H., S. J. Kim, Y. C. Park, Y. Hwang, O. J. Yoo, and Y. C. Kim. 1994. Nucleotide sequence of the penicillin G acylase gene from Bacillus megaterium and characteristics of the enzyme. Misainmurhag. Hoiji. 32:215-221.
Kikuchi, Y., H. Itaya, M. Date, K. Matsui, and L. F. Wu. 2009. TatABC overexpression improves Corynebacterium glutamicum Tat-dependent protein secretion. Appl. Environ. Microbiol. 75:603-607.

Kolkman, M. A. B., R. van der Ploeg, M. Bertels, M. van Dijk, J. van der Laan, J. M. van Dijl, and E. Ferrari. 2008. The twin-arginine signal peptide of Bacillus subtilis YwbN can direct either Tat- or Sec-dependent secretion of different cargo proteins: Secretion of active subtilisin via the B. subtilis Tat pathway. Appl. Environ. Microbiol. 74:7507-7513.

Kunst, F., N. Ogasawara, I. Moszer, A. M. Albertini, G. Alloni, V. Azevedo, M. G. Bertero, P. Bessières, A. Bolotin, S. Borchert, R. Borriss, L. Boursier, A. Brans, M. Braun, S. C. Brignell, S. Bron, S. Brouillet, C. V. Bruschi, B. Caldwell, V. Capuano, N. M. Carter, S. K. Choi, J. J. Codani, I. F. Connerton, and A. Danchin.. 1997. The complete genome sequence of the gram-positive bacterium Bacillus subtilis. Nature 390:249-256.

Li, W., X. Zhou, and P. Lu. 2004. Bottlenecks in the expression and secretion of heterologous proteins in Bacillus subtilis. Res. Microbiol. 155:605-610.

Müller, J. P., Z. D. An, T. Merad, I. C. Hancock, and R. C. Harwood. 1997. Influence of Bacillus subtilis phoR on cell wall anionic polymers. Microbiol. 43:947-956.

Pop, O., U. Martin, C. Abel, and J. P. Müller. 2002. The twin-arginine signal peptide of $\mathrm{PhoD}$ and the Tat $\mathrm{A}_{\mathrm{d}} / \mathrm{C}_{\mathrm{d}}$ proteins of Bacillus subtilis form an autonomous Tat translocation system. J. Biol. Chem. 277:3268-3273.

Sambrook, J., and D. W. Russell. 2001. Molecular Cloning: A Laboratory Manual. 3rd ed. Cold Spring Harbor Laboratory Press, New York, NY.

Smith, T. J., S. A. Blackman, and S. J. Foster. 2000. Autolysins of Bacillus subtilis: Multiple enzymes with multiple functions. Microbiol. 146:249-262.

Tjalsma, H., H. Antelmann, J. D. H. Jongbloed, P. G. Braun, E. Darmon, R. Dorenbos, J.-Y. F. Dubois, H. Westers, G. Zanen, W. J. Quax, O. P. Kuipers, S. Bron, M. Hecker, and J. M. van Dijl. 2004. Proteomics of protein secretion by Bacillus subtilis: Separating the "secrets" of the secretome. Microbiol. Mol. Biol. Rev. 68:207-233.

Tjalsma, H., A. Bolhuis, J. D. H. Jongbloed, S. Bron, and J. M. van Dijl. 2000. Signal peptide-dependent protein transport in Bacillus subtilis: A genome-based survey of the secretome. Microbiol. Mol. Biol. Rev. 9:515-547.

van Dijl, J. M., P. G. Braun, C. Robinson, W. J. Quax, H. Antelmann, M. Hecker, J. Müller, H. Tjalsma, S. Bron, and J. D. H. Jongbloed. 2002. Functional genomic analysis of the Bacillus subtilis Tat pathway for protein secretion. J. Biotechnol. 98:243-254.

Wanarska, M., J. Kur, R. Pladzyk, and M. Turkiewicz. 2005. Thermostable Pyrococcus woesei $\beta$-D-galactosidase-high level expression, purification and biochemical properties. Acta Biochim. Pol. 52:781-787.

Westers, L., D. S. Dijkstra, H. Westers, J. M. van Dijl, and W. J. Quax. 2006. Secretion of functional human interleukin-3 from Bacillus subtilis. J. Biotechnol. 123:211-224.

Xia, Y., W. Chen, X. Y. Fu, H. Zhang, S. Yang, and X. L. Ding. 2005. Construction of an integrative food-grade expression system for Bacillus subtilis. Food Res. Int. 38:251-256.

Yuan, G., and S. L. Wong. 1995. Regulation of groE expression in Bacillus subtilis: The involvement of the $\sigma^{\mathrm{A}}$-like promoter and the roles of the inverted repeat sequence (CIRCE). J. Bacteriol. 177:5427-5433. 